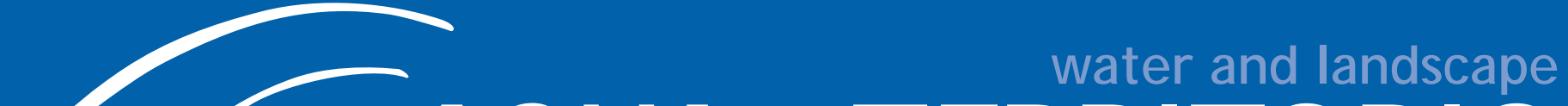 AGUAYTERRIORIO
}

\section{Tierra y Agua: efectos culturales por la construcción de la hidroeléctrica en los pueblos indígenas nahuas de Zongolica, Veracruz}

\author{
Earth and Water: Cultural Effects among Indigenous Nahua People Arising \\ from the Construction of a Hydroelectric Power Plant in Zongolica, Veracruz
}

Martha Inés Flores-Pacheco

Universidad Veracruzana. Xalapa, México. martflores@uv.mx

\begin{abstract}
Resumen - El artículo que se presenta tiene como objetivo exponer el caso concreto del pueblo indígena nahua afectado por la construcción de una presa hidroeléctrica en Zongolica, Veracruz. El caso muestra cómo, una vez más, el estado mexicano realiza este tipo de obras sin cumplir con los debidos procesos de consulta y participación de la población afectada. Más aún, sin tomar en cuenta los perjuicios de la obra en el territorio y la cultura de los indígenas de la zona. La metodología utilizada en la investigación del caso es inductiva, basada en el método etnográfico y en la observación participante. Se recupera información de entrevistas realizadas en el trabajo de campo y se utilizan fuentes hemerográficas. Los resultados de la investigación muestran que una constante en este tipo de obras es la violación a los derechos de los pueblos indígenas; así como las irregularidades cometidas reiteradamente por las instituciones de gobierno que se hacen cargo de estos procesos de consulta, indemnización y reacomodo de las poblaciones. La investigación documenta el proceso seguido por el gobierno mexicano en la afectación de los indígenas nahuas de Veracruz y la forma en que se está alterando y será modificado su modo de vida y su cultura. A lo largo del texto se abordan preguntas como icuáles son los efectos (sociales, culturales y territoriales) de estas grandes obras en la vida de los pueblos indígenas? ¿Por qué estas obras no toman en cuenta los derechos de los pueblos indígenas ya consagrados en la legislación nacional e internacional? y ¿cómo pueden los pueblos indígenas obligar al estado mexicano a respetar sus derechos?

Abstract - This article deals with the construction of a hydroelectric dam in Zongolica, Veracruz and its effects on the local indigenous Nahua people. The case shows how, once again, the Mexican State carries out these public works projects without complying with the due processes of consultation and participation of the affected population. And, what's more, the State does so without taking into account the harmful effects of the projects in the territory and culture of the indigenous people of the area. The methodology used in the investigation is inductive, based on an ethnographic approach, participant observation techniques, field interviews and newspaper sources. The results show that these types of projects consistently violate the rights of indigenous peoples. A series of irregularities are repeatedly committed by government institutions that are responsible for implementing the processes of consultation, compensation and resettlement of the affected populations. The research documents the procedures used by the Mexican government, their impact on the indigenous Nahua people of Veracruz and the way these measures are altering and will be modifying the way of life and culture of the indigenous people. The following questions are posed: what are the (social, cultural and territorial) effects of these vast projects on the lives of the indigenous peoples? Why do they disregard the rights of the indigenous peoples that are already enshrined in national and international law? How can the indigenous peoples compel the Mexican State to respect their rights?
\end{abstract}

Palabras clave: pueblos indígenas, agua, cultura, derechos y territorio Keywords: indigenous peoples, water, culture, rights and territory 


\section{INTRODUCCIÓN}

Desde el siglo pasado, el tema de la construcción de presas hidroeléctricas y de almacenamiento está vigente en los planes de desarrollo del estado mexicano, que impulsa estas grandes obras argumentando que es por causa de "utilidad pública" ${ }^{1}$ y que provocan la destrucción de los ecosistemas, el patrimonio histórico y cultural, pero principalmente, afectan los territorios, la territorialidad, la vida cultural y los derechos de los pueblos indígenas. Por ello, desde hace décadas, a lo largo y ancho de México, instituciones académicas y organismos no gubernamentales, entre otros, se dedican a difundir, reclamar, asesorar, estudiar y defender los casos que se han ido presentando en varias entidades del país. Para conocer un estudio de caso, se expondrá el proceso de construcción de una presa hidroelectrica en la Sierra de Zongolica, perteneciente al estado de Veracruz.

México tiene una larga experiencia en la construcción de presas hidroeléctricas y represas. Hoy en día hay en todo el territorio 4.200, a las que habría que añadir treinta nuevas que se tiene planeado erigir sobre territorios indígenas para antes del 2017. Esto significa que los gobiernos de turno, independientemente de su filiación política, continúan construyéndolas bajo un esquema que transgrede tratados internacionales y nacionales relacionados a los pueblos indígenas, violando sus derechos humanos, culturales, territoriales, políticos y sociales, aprovechando su ignorancia y dejándolos en desventaja ante las grandes empresas, originando graves violaciones.

El caso de la presa de la Sierra de Zongolica es un proyecto hidroeléctrico en Veracruz que comenzó a construirse en 2008, con una capacidad de almacenamiento de agua de $194.430 \mathrm{~m}^{3}$. Se encuentra ubicado en la zona centro suroeste del estado, en la denominada Sierra Negra de Zongolica, que forma parte de la región natural de las Grandes Montañas, en la cola del embalse del río Apatlahuaya. El proyecto hidrológico está afectando directamente a tres municipios indígenas: el municipio de Mixtla con la construcción del embalse; el de Texhuacán con la edificación de la cortina, el túnel que la une al embalse y el pozo de oscilación, y el municipio de Zongolica con la tubería de presión, casa de máquinas y la subestación.

Este artículo forma parte de la investigación realizada durante los años 2012-2014 en el Instituto de Antropología de la Universidad Veracruzana intitulada "Políticas sociales y Pueblos Indígenas: turismo y patrimonio histórico, cultural y natural de la Región de las Altas Montañas, Veracruz". En su realización hubo tres periodos de trabajo de campo a lo largo de 2013 y se llevaron a cabo 40 entrevistas a los pobladores de la región sobre diferentes contenidos de la investigación. En particular, sobre el tema de la presa, se entrevistó a diez personas y se realizó un recorrido por la zona en la que ya se pudo observar la destrucción del bosque, el entubado de los ríos y los desastres naturales que está provocando en tres de los municipios directamente afectados: Mixtla de Altamirano, Texhuacán y Zongolica. A esto se agrega el daño cultural, social y político hacia el pueblo indígena nahua.

\footnotetext{
1 Scott, 2012, 10 .
}

La construcción de la presa ha movilizado a los pobladores y a algunos organismos no gubernamentales que han puesto en la agenda estatal los graves problemas que implica llevar a cabo esta obra. Es importante mencionar que también en otros varios estados del país, pobladores indígenas están realizando movilizaciones a causa de la construcción de presas en sus territorios².

Estos proyectos muestran un Estado que ignora y continúa promoviendo estas construcciones obedeciendo a un modelo de desarrollo económico que contribuye a seguir marcando la polaridad social y económica en México, pues se incentiva el enriquecimiento de los inversionistas privados, mientras que se deja en la miseria a los dueños de los territorios al quedarse sin tierras para sembrar, alejados de sus ancestros y sin una vivienda digna, despojándolos finalmente de sus formas de vida. En ocasiones, estas empresas prometen un cambio de vida a los pobladores, pero en la mayoría de los casos no cumplen y faltan impunemente a su palabra, violando toda clase de leyes nacionales e internacionales 3 . Es por ello que a lo largo del texto se irán abordando preguntas como ¿cuáles son los efectos (sociales, culturales y territoriales) de estas grandes obras en la vida de los pueblos indígenas? ¿Por qué estas obras no toman en cuenta los derechos de los pueblos indígenas ya consagrados en la legislación nacional e internacional? y ¿cómo podrían los pueblos indígenas obligar al estado mexicano a respetar sus derechos?

\section{Referencias conceptuales}

El tema de las relaciones entre cultura, sociedad y espacio, así como el tema del territorio, ha sido abordado por la antropología desde sus orígenes. Sin embargo, el lugar que ha ocupado el territorio en el análisis de los fenómenos antropológicos ha variado en función de las diversas perspectivas teóricas. En general, las distintas tradiciones antropológicas han enfatizado las interrelaciones y vínculos de las estructuras y procesos locales, regionales y globales, pero sobre todo las apropiaciones materiales del espacio, así como la representación mítica del territorio y uso del espacio por parte de los sujetos sociales. Trabajos más recientes han reconocido que la naturaleza y el espacio son construcciones sociales en permanente cambio y que varían según la especificidad cultural y los contextos históricos en que se insertan

2 Hay varios proyectos de presas en el país con estudios de factibilidad técnica y financiera concluidos, y que han generado amplios movimientos sociales de resistencia. Su eficacia ha paralizado, hasta hoy, las obras programadas en San J uan Tetelcingo y La Parota (CFE/ Guerrero), Paso de la Reina y Paso Ancho (CFE/Oaxaca), Las Cruces (CFE/ Nayarit), Boca del Monte/Tenosique, Chicoasén II/ Copainalá (CFE/ Chiapas), Bicentenario (Conagua/ Sonora), La Yesca (2012, CFE/ Jalisco), Río Moctezuma (2014, CFE/ Hidalgo) y Francisco J. Múgica (2012, Conagua/ Michoacán) y proyectos factibles de escala menor o mediana con concesiones a empresas privadas en los ríos de Chiapas, Puebla y Veracruz (Scott, 2012, 10).

3 El desarrollo de la energía hidroeléctrica y de abasto de agua por construcción de presas en México ha dado lucrativas ganancias a unos cuantos, a costa de la pérdida de vidas, tierras y modos de sustento, mediante la violación de leyes nacionales e internacionales. Las afectaciones no solo incluyen la falta de vivienda digna y dotación de tierras, sino problemas de salud física y mental, la disminución de cultivos y alimentación, experiencias individual y colectiva de tensión, nervios, ansiedad, temor, depresión y tristeza profunda y duradera, así como problemas sociales, resultado de vivir en un clima de continuas sospechas, estigmatización y temor, y la pérdida de sitios sagrados y culturales, como cementerios y lugares de oración (Sánchez y Méndez, 2012). 
los suj etos 4 . Dentro de este marco han adquirido renovada importancia los conceptos de espacio, |ugar $^{5}$, territorio y paisaje, que desde la antropología, la arqueología y la geografía ${ }^{6}$, han tendido a ser definidos más en función de la cultura y de la sociedad que solo desde los aspectos físicos o geomorfológicos.

De este modo, las relaciones entre cultura, territorio y sociedad son creaciones históricas y sociales y no el resultado de factores "naturales" o determinaciones culturales. El territorio, según Giménez ${ }^{7}$, conjuga distintas dimensiones, pues constituye, por una parte, un "espacio de inscripción de la cultura", lo que equivale a una de sus formas de objetivación; sirve como marco 0 área de distribución de instituciones y prácticas culturales espacialmente localizadas; y, por último, puede ser apropiado subjetivamente, como objeto de representación y de apego afectivo, como símbolo de pertenencia socioterritorial.

El territorio es el ámbito espacial que histórica y culturalmente ha sido apropiado por un pueblo, pero también es un espacio que culturalmente se construye a través del tiempo por lo que es definido como territorio. En el espacio del territorio hay tradiciones, memoria histórica, costumbres, ritos y distintas formas de organización social que lo conforman como territorio cultural. La territorialidad entendida como un fenómeno colectivo, es el resultado de la múltiple articulación históricamente establecida entre la naturaleza y la sociedad en contextos de interacción específicos. Por otra parte, la territorialidad simbólica adquiere un matiz o un carácter especial que la antropología y, en particular, la etnología tratan bajo determinados enfoques, lo que hace que estas no solo difieran, sino que también se alejen de las categorías geográficas o político-administrativas, aunque estos parámetros no dejan de relacionarse con el territorio. La territorialidad simbólica se vincula con categorías de representaciones territoriales estructuradas, articuladas y en acuerdo con una lógica interna que es propia de las culturas. Los territorios simbólicos están marcados por la cosmovisión, por las prácticas rituales, la mitología y los lugares sagrados que llegan a ser emblemas territoriales e identitarios y esto en sí es el territorio cultural, su espacio es la urdimbre de representaciones, concepciones y creencias de profundo contenido emocional ${ }^{8}$.

La idea de des-territorialización provocada por megaproyectos alude al supuesto desanclaje de la cultura respecto de su vínculo con el territorio como una resultante de los procesos de cambio operados a través de la globalización, en un escenario que tiene como referencia dos fenómenos paradigmáticos: a) La reformulación del papel del Estado como núcleo ordenador de la morfología social, con la puesta en crisis de toda una serie de nociones asociadas a la identidad nacional y su anclaje en una territorialidad estatal. b) La anulación tecnológica de las distancias de tiempo y espacio, que habilitó la problemática de las diferentes escalas involucradas en el cambio social a través de la articulación de lo local, lo nacional y lo global ${ }^{9}$.

\footnotetext{
4 Descola, 2001.

5 De Certeau, 2000

6 Hoffmann y Salmerón, 1997

7 Giménez, 2000, 29-30.

8 Barabas, 2001, 16-18.

9 Haesbaert, 2004, 400.
}

Por lo tanto, en este análisis, el territorio es el tema medular y, desde la mirada antropológica, se considera como una totalidad interdependiente, en el que se entrelazan elementos y fenómenos históricos, semióticos, culturales y naturales.

En primer lugar, hay que considerar que el territorio para la mayoría de los pueblos indígenas de México y de origen mesoamericano -y por ende para los nahuas de Zongolica- es sagrado; contiene referentes simbólicos y elementos en común. Mantienen una relación estrecha con el universo, el territorio, la geografía y el inframundo. Velasco ${ }^{10}$ agrega además que existe una relación entre el hombre, los dioses, las deidades y el territorio (naturaleza), desde lo histórico, cultural y social, a través de por lo menos cinco elementos: 1) Concepción sagrada del mundo; 2) Imagen territorial y sus límites; 3) Reconstitución étnica a partir de la organización familiar; 4) Memoria histórica; y 5) La soberanía comunitaria en la que reside la responsabilidad del territorio cedido por los dioses al hombre, por lo que tiene la obligación de renovar el vínculo deidades-hombres. Esta concepción del mundo se encuentra vinculada con la identidad y la territorialidad de sus habitantes, así como con algunos rituales que se practican actualmente.

En segundo lugar, el territorio es una construcción social, resultado de diversas formas de apropiación del espacio del que participan diversos actores, como se mencionó líneas arriba. Estas múltiples apropiaciones se nutren de la cultura, de la memoria y las experiencias sociales surgidas de los conflictos territoriales o agrarios, así como de los procesos históricos, resultado de las relaciones con el Estado nacional.

De acuerdo a Rodríguez ${ }^{11}$, en la concepción territorial de los nahuas de la Sierra de Zongolica existe una metáfora cósmica de la posición arriba-abajo, donde la superficie terrestre (Tlaquitepatl), la tierra y el señor del subsuelo, dueño de los animales y la vegetación silvestre, tlalokan, se encuentran en constante diálogo. Esta geografía simbólica contiene lugares que representan algún hecho social o cultural. "En la religiosidad de los nahuas de Zongolica (...) destaca la existencia de tlalokan como un ámbito subterráneo de riqueza y abundancia que desemboca en las cuevas de las montañas y es morada de los tlalokes, deidades relacionadas con las Iluvias, los animal es silvestres y la vegetación" 12 .

En tercer lugar, los territorios indígenas se han visto afectados por el impulso de proyectos de desarrollo (turismo, infraestructura, servicios, entre otros) con el interés de potenciar recursos para mejorar el nivel de vida de las comunidades étnicas y rurales. Pero en estos proyectos ¿qué papel se le ha asignado a la cultura y al territorio como patrimonio cultural de las comunidades? En la mayoría de los casos la territorialidad étnica es ignorada y violentada, por ello es uno de los temas prioritarios en la plataforma de reivindicaciones indígenas, no únicamente en su condición de derecho colectivo indispensable, sino como una verdadera dimensión existencial de cada pueblo.

Y en cuarto lugar -y de acuerdo a la reflexión y análisis de los investigadores y estudiosos del tema- se considera que hay

10 Velasco, 2002, 143-145.

11 Rodríguez, 2003.

12 Ibidem, 224 
un vacío en la legislación en lo relativo al territorio como un bien cultural y como un derecho colectivo, que junto con las demandas por la autonomía y autodeterminación de los pueblos indígenas, se plantea el reconocimiento de derechos de exclusividad territorial, señalando que poseen una relación intrínseca con el espacio, relación culturalmente mediada que tendría consecuencias sobre sus identidades y formas de vida.
Mapa 1. Ubicación de la región de las montañas en el estado de Veracruz, México
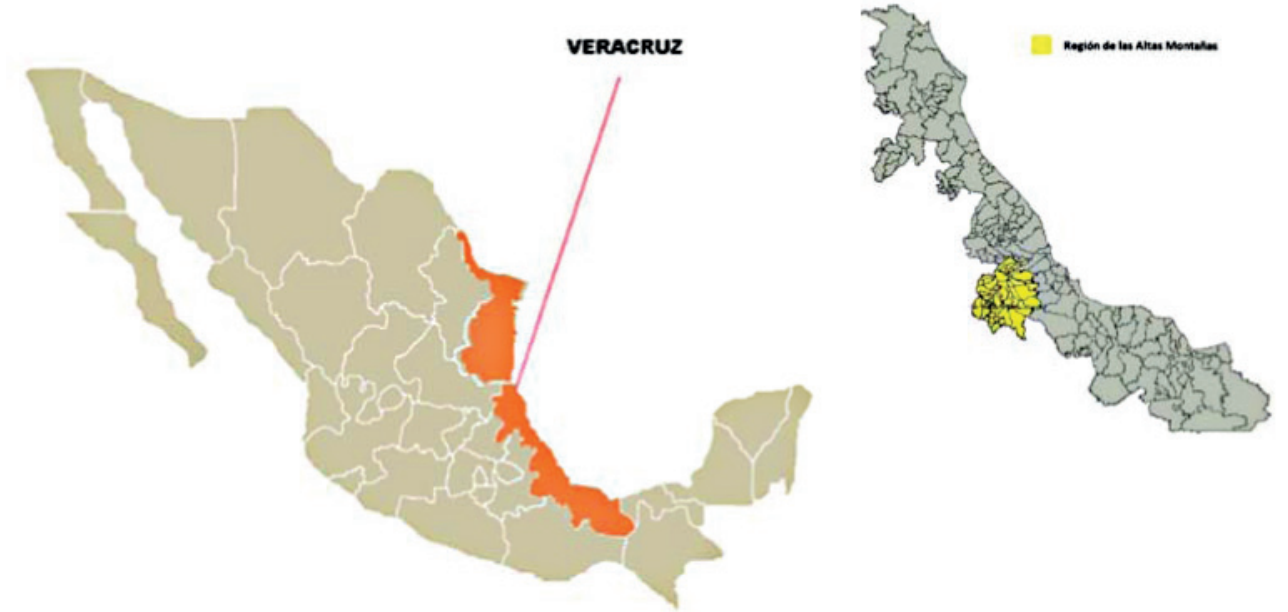

Fuente: http:// es. wikipedia.org/ wiki/ Zongolica (consulta del 18 de agosto de 2013).

\section{La región de Zongolica, Veracruz-Llave}

México se encuentra dividido en 32 entidades federativas, el estado de Veracruz-Llave es una de ellas, comprende una larga franja de tierra de bordes irregulares delimitada por el mar y por montañas. Tiene una extensión territorial de $71.699 \mathrm{~km}^{2}$ y una población de 7.643.194 habitantes, al norte, colinda con Tamaulipas, al sur con Oaxaca y Chiapas, al oriente con el Golfo de México, al poniente con San Luis Potosí, Hidalgo y Puebla, y al sureste con Tabasco ${ }^{13}$. La región de Zongolica forma parte de la Sierra Madre Oriental, colinda al este con la Llanura Costera del Golfo; al oeste con la vertiente occidental de la misma sierra; al norte con el Pico de Orizaba y el Cañón de Río Blanco; y al sur con la Sierra Mazateca de Oaxaca. Cuenta con una superficie aproximada de 1.714,9 km²; abarcando 14 municipios. En esta región viven principalmente nahuas asentados en valles, cumbres y cañadas que conforman las tres zonas climáticas y ecológicas.

La construcción de la presa afecta a dos regiones de la sierra: la zona templada - donde se ubica Zongolica con 41.923 habitantes-y la zona baja de los municipios de Mixtla de Altamirano con 10.387 habitantes y el de Texhuacan con 5.292 habitantes. Las comunidades que han sido afectadas por la presa son las siguientes: en Texhuacan, Palulca, Equimititla, Apanga, Atzingo, Tepetlapa; en Mixtla de Altamirano, Xometla, Xala y Xochitla; y en Zongolica, Macuilca, Comalapa, Zomajapa, Piedras Blancas, Acontla, Puente Porras, Ocotitla, Apanga, Tonalixco, Ixcohuapa, Tonacalco y Zacatal Chico.

El cultivo del maíz no solo les es indispensable para la subsistencia, también lo es para crear y recrear sus concepciones ancestrales pues la obtención de los frutos naturales se concibe como una negociación entre los hombres (cultura) y la naturaleza, cuyos dueños son deidades o dioses. Por esto recurren a los ritos propiciatorios, a través de los cuales se alcanza el favor y los dones de la naturaleza.

\footnotetext{
13 INEGI, 2010.
}

\section{BaLANCE SObRe La CONSTRUCCIÓN DE PROYeCtOS HIDROELÉCTRICOS EN TERRITORIOS DE LOS PUEBLOS INDÍGENAS}

El proceso de construcción de presas hidroeléctricas en México comienza a mediados del siglo XX y se acrecienta con la llegada y participación de la inversión privada a partir de los años noventa. El primer período de desplazamientos masivos de pueblos indígenas ocurre con la construcción de la presa Miguel Alemán (ubicada entre los estados de Oaxaca y Veracruz) en 1950, con un desalojo forzado de 22.000 mazatecos. Este episodio para los inconformes que rehusaron perder la tierra- finalizó con el incendio de sus casas. En 1972, 20 años más tarde, se inicia la construcción de la presa Cerro de Oro en el estado de Oaxaca, que desplazó a 26.000 indígenas chinantecos. Han pasado 40 años y los afectados continúan un proceso legal para obtener indemnizaciones más justas sobre su territorio ${ }^{14}$.

Una década después, entre 1981-1986, se construyó en el estado de Guerrero la presa El Caracol. Durante este proceso quedaron afectados 11 poblados nahuas, solo la comunidad Balsas Sur pudo resistir temporalmente en su territorio hasta que la Comisión Federal de Electricidad (CFE), institución encargada de promover la obra, inundó la comunidad sin previo aviso. Aun así, Balsas Sur continuó con el proceso legal contra la CFE y exitosamente logró un amparo ex post facto, esto es, después de cometida la afectación ${ }^{15}$.

Conseguir el amparo permitió a los nahuas que los medios masivos de comunicación e investigadores tomaran en cuenta el caso y lo documentaran, estableciéndose además un precedente legal importante ya que sirvió para que los nahuas se asesoraran y pudieran denunciar en el ámbito internacional otro desplazamiento forzado y las afectaciones socio-ambientales que ocasionaría la edificación de la presa El Caracol. Con ello se consigue cuestionar la política gubernamental para la construcción de presas en todo el país. De igual modo, como la fuente de financiamiento para la construcción de la presa era el Banco Mundial

14 HIC-AL, 2012, 1. HIC-AL, 2010, 7. Presa Cerro de Oro, 35 años sin justicia. 15 HIC-AL, 2010, 12. HIC-AL, 2012, 2. 
Tierra y Agua: efectos culturales por la construcción de la hidroeléctrica en los pueblos indígenas nahuas de Zongolica, Veracruz

(BM), este modificó sus lineamientos, obligando a la Comisión Federal de Electricidad a crear la Gerencia de Desarrollo Social (GDS), órgano normativo regulador y coordinador para atender los problemas políticos y sociales derivados de los proyectos hidroeléctricos, para poder obtener financiación en la construcción de las hidroeléctricas Zimapán (en los estados de Querétaro e Hidalgo) y Aguamilpa (en Nayarit) ${ }^{16}$.

En consecuencia, en la última década del siglo XX, para la construcción de la presa de Aguamil pa se contrató a un equipo de antropólogos y sociólogos, cuya tarea fue "convencer" a los huicholes o wixarikas para que vendieran sus tierras y se movieran hacia la ribera de lo que sería el embalse, formando pequeñas localidades. Al principio ellos fueron los responsables del manejo de la presa, controlando los medios de transporte, tanto terrestres como lacustres. Por vez primera quedó de manifesto que dentro de las afectaciones por la construcción de la presa los lugares sagrados de sus ancestros quedarían bajo el agua, por lo que la CFE aceptó que se llevaran a cabo una serie de rituales para pedir permiso a los dioses a fin de poder moverse de sus moradas. Con el cambio de residencia los pueblos wirrarikas transformaron su forma y sus condiciones de vida. Aunque aparentemente mejoraron sus niveles de bienestar, perdieron parte de su identidad cultural al dejar a los dioses enterrados debajo de la presa.

Hoy en día se está denunciando en la prensa nacional que el agua de la presa ${ }^{17}$ está contaminada. De acuerdo con señalamientos del presidente de la Fundación de la Defensa wixarika de Nayarit, Braulio Muñoz Hernández, esto se debe al derrame de aguas negras al río Santiago, provenientes del estado de Jalisco, que afecta directamente a 10.000 indígenas coras y huicholes que habitan en 19 comunidades aledañas a la presa. Esto ha llevado a solicitar a la Conagua (Comisión Nacional del Agua) que intervenga para tratar el afluente. A esta situación se agrega la denuncia pública presentada por la comunidad indígena de Santa Catarina Cuexcomatitlán Tuapurie, territorio wixarika al norte de Jalisco, mediante una carta firmada en asamblea de la comunidad el 30 de abril del 2006 por las autoridades tradicionales, la cual señala que la presa de La Yesca:

"perjudica directamente a nuestros compañeros wixaritari, despojándolos de sus tierras, y a los ríos sagrados de Santiago y Bolaños que pasan por nuestros territorios por lo que los consideramos sagrados, no solo porque de ellos vivimos, sino porque el agua es la sangre que corre por nuestra madre tierra"18.

16 Tribunal Permanente de los Pueblos, 2012, 3. HIC-AL, 2010, 12. "...Muchas de las decisiones generadas en el territorio estudiado han dependido de como se ha percibido el desarrollo por el gobierno mexicano, los funcionarios consideran las hidroeléctricas como detonadores económicos por los beneficios que llevan a las regiones" (Iracheta-Cenecorta y Gómez, 2014, 45).

$17 \mathrm{http}: / /$ www. sinembargo. mx (2012).

18 www. puebloindígena.com/noticias/...de...presa-aguamilpa, 2013. Para la comunidad wixarika, los ríos son la sangre de la tierra. Para el estado mexicano son fuente de riqueza, son negocio, y si uno envenena la sangre y la obstruye ¿qué pasa con los dioses y el territorio? Como se puede observar, las acciones de la CFE, a pesar de haber aceptado la participación de la población afectada y haber realizado programas de desarrollo orientados a la reconstrucción de la base productiva, resultaron insuficientes, inadecuadas e incompletas.
En 2007, en San J uan Tetelcingo -Guerrero-la CFE comenzó la construcción de un proyecto hidroeléctrico sin informar a los nahuas del Alto Balsas que sus comunidades quedarían anegadas por el embalse. Para su defensa y protección, los nahuas conformaron el Consejo de Pueblos Nahuas del Alto Balsas (CPNAB), que sostuvo la consigna: De nikan para techkixtiske, xtopa techmiktiske (para sacarnos de aquí, primero tendrán que matarnos). EI CPNAB condujo exitosamente a 37 comunidades para detener la obra ${ }^{19}$.

Otro caso que alteró la vida y los lugares sagrados de los pueblos indígenas fue el de los mayo de Huites, Sinaloa, que en 1996 fue afectado por la construcción de la presa Luis Donaldo Colosio. En este lugar se llevó a cabo el Primer Encuentro de Poblaciones Indígenas Desplazadas por la Construcción de Presas, donde elaboró la Declaración de Huites: " ... por ignorarlos en la planeación de las obras; por excluirlos de la repartición de los posibles beneficios que generan; por violentar sus territorios y por no apoyar en la preservación de su cultura" 20 . La Declaración de Huites señala directamente al Gobierno Federal como el principal responsable por permitir que otros violen sus derechos con el fin de favorecer grandes intereses privados. El problema de los mayos de Huites es que fueron arrancados de sus antepasados, de sus casas, de sus muertos, de su centro ceremonial y de su entorno natural con la construcción de la presa, aunque ellos siguen en lucha para no perder su legado cultural y social ${ }^{21}$.

Los casos anteriores son solo un ejemplo de la problemática que existe en México. Pueblos indígenas mayos, mazatecos, chinantecos, tzotziles, nahuas, zoques, pames, otomíes, yaquis, wirarikas, coras, zapotecos, sólo por mencionar algunos, son afectados por estas obras. Por ello, antropólogos han calificado esta práctica como etnocida22. A la fecha, se calcula que han sido desplazadas en México forzosamente más de ciento ochenta y cinco mil personas ${ }^{23}$.

Pese al discurso desarrollista que acompaña estos megaproyectos, el despojo de tierras y propiedades por estas obras ha provocado un mayor empobrecimiento de los pueblos indígenas y la destrucción de su cultura ${ }^{24}$.

19 Tribunal Permanente de los Pueblos, 2012, 3. HIC-AL, 2010, 12.

20 Ibidem.

21 Pérez, 1996.

22 Barabas y Bartolomé, 1992, 7.

23 Ibidem, 9 .

24 "Hoy las presas hidroeléctricas e hidráulicas son ejemplos de la imposición de una estrategia energética sui generis del Estado (y sus socios), pero también símbolos de un modelo de desarrollo agresivo, extractivo y violador de derechos fundamentales de las comunidades, por los desplazamientos en condiciones discrecionales e injustas que generan (...) las presas representan un género de proyectos de inversión y despojo de gran escala que desplazan a ciudadanos y poblados de manera arbitraria, sin concederles participaciones en los negocios. Este nuevo género, que no merece nuestro respaldo bajo las reglas actuales, también abarca concesiones mineras, carreteras de cuota (...), campos eólicos, nuevos desarrollos petroleros, puertos industriales y desarrollos turísticos e inmobiliarios. El patrón histórico indica que los desplazados de hecho se convierten, sin consultas previas ni opciones dignas, en víctimas del "progreso". Y el anuncio de cada proyecto detona un proceso de movilizaciones entre las pocas organizaciones sociales regionales que se oponen al modus operandi tradicional de estos megaproyectos, y quienes, últimamente, se aprovechan para bien de las redes sociales digitales para difundir y ampliar cualquier campaña" (Scott, 2012, 10). 


\section{EL INICIO deL PROYeCto Y EL DESPOJ O de tieRras en Zongolica, VeracruZ}

Para la presa de la región de Zongolica se vuelven a repetir las mismas irregularidades y violaciones a los derechos de los pueblos indígenas. Uno de ellos establece que los pueblos tienen el derecho de ser consultados e informados de las obras que afecten a su territorio. Sin embargo, testimonios de la población señalan que:

"A la gente nunca la consultaron para la instalación de esta represa. Nunca se les informó ni se les pidió su opinión, si estaban de acuerdo 0 no. Únicamente, allá por el 20080 2009, nos enteramos [de] que gente de fuera estaba adquiriendo terrenos, hectáreas de terreno, y nunca supimos de qué se trataba. La gente accedió a esa compra venta de terrenos, los vendió, pero ahora que se dieron cuenta del uso que le están dando a esas tierras, empieza la gente a conmoverse y a incomodarse" (María Cira, de Toaltepeyólotl) ${ }^{25}$.

Para la apertura de esta represa tuvieron que derribar un puente de aproximadamente doscientos años de antigüedad, incomunicando a poblados, como lo menciona María Cira:

"Ahora les abrieron una brecha por otro lado. Antes, sobre ese
puente, pasaban con bestias su carga. Ahora no pueden pasar bes-
tias y la gente tiene que cargar ella misma. Las comunidades afec-
tadas por la destrucción del puente de piedra pertenecen al muni-
cipio de Mixtla de Altamirano, la más cercana y la más afectada se
llama Xometla" (María Cira, de Toaltepeyólotl) ${ }^{26}$.

En el oficio S.G.P.A./ DGIRA.DG.6125.09, de la Secretaría del Medio Ambiente y Recursos Naturales (Semarnat), se indica que la dependencia entregó el permiso para la instalación del proyecto en octubre de 2009, con una vigencia de tres años para la construcción y de 25 años para su operación. Las afectaciones estaban en torno a los ciento doce mil metros cuadrados de bosques de pino y encino, de tierras de cultivo y de uso pecuario. La autorización de uso limitado fue entregada por la misma Semarnat siete meses después de que rechazara un primer permiso a la empresa ${ }^{27}$.

La empresa Electricidad del Golfo S de RL de CV y el corporativo estadounidense Conduit Capital Partnes (LLC) señalaban que, además de las reuniones informativas, celebraron consultas públicas en la región. La primera de ellas fue en febrero de 2009, es decir, más de un año antes de que empezaran los trabajos de construcción de la presa. Cuando se le preguntó a un representante de la empresa ien qué consistió esa consulta pública? expresaba que se trataba de una invitación a la población, donde se les hacía un resumen de la manifestación de impacto ambiental; de las condicionantes del resolutivo ambiental y de los permisos

\footnotetext{
25 http:/ / zapateando. wordpress.com (2013).

26 Ibidem.

27 Tanto las empresas como las instituciones de gobierno no están interesadas en informar, tampoco se preocupan por consultar y mucho menos en dar a conocer a las poblaciones afectadas los proyectos a implementar. Los pobladores manifiestan que no saben nada sobre el proyecto. Sin embargo, la visión empresarial que ignora y desvaloriza a los pueblos indígenas se ilustra con las declaraciones de un ingeniero de la empresa que considera que son ellos (la población) quienes no han tenido interés en acercarse.
}

que ya se habían otorgado o estaban en proceso. Consideraban que una consulta pública no significaba tener la anuencia de la población, sino que podían escuchar sobre el proyecto, podían hacer preguntas, podían inconformarse "con las autoridades 0 con nosotros" para poderles responder. Una consulta pública no era llegar a pedir su autorización pues esta la daba la autoridad28.

Actualmente, la construcción de la presa está a más de la mitad. Al mismo tiempo, la población cada vez está más informada de las afectaciones que representa para su territorio, lo que está dando lugar a protestas y movilizaciones en la región.

En la sierra de Zongolica, los comentarios de los habitantes son contundentes:

"creímos en ellos, pero todo se está secando, todo es incierto, todo, como en todo el país fue a base de engaños, es como morir esperando un futuro que nunca llegará. Los que han luchado por la no construcción se han desilusionado ante tanta mentira, la injusticia se nota, pero las autoridades locales lo niegan". Doña Ada cuenta que, cuando ella se muera, no será enterrada en su tierra "pues ya quedará tapada por las aguas" (Filadelfia Lara, Palulca) ${ }^{29}$.

Tampoco les pagarán sus árboles frutales, ni su café y menos les entregarán pronto la indemnización. Algunos representantes de la empresa se valieron de engaños utilizando prácticas rituales de los pobladores para favorecer la causa de la obra. Las comunidades nahuas realizan un ritual llamado xochitlalli, el cual tiene como finalidad pedir permiso a la tierra para propiciar el crecimiento adecuado de la cosecha, y en tiempo de sequía se pide agua. Uno de los habitantes de esta región explicó que:

"para lograr la construcción de la represa y con la finalidad de venir a introducirse a la cultura nahua, se mandaron a hacer xochitlallis, pero los pobladores de la sierra de Zongolica lo vemos mal; no es correcto porque esto representa una burla hacia la madre tierra".

Agregó:

"¿Cómo es posible que le pidas permiso para destruirla? Casi en su totalidad vas a destruirle el rostro, la vas a herir, la vas a lastimar, la vas a perforar, vas a acabar con su ropaje, vas a acabar con sus venas, que son los ríos; vas a desviar el flujo de su sangre, ¿qué es el agua?, solo para servir a intereses económicos, para servir a proyectos que atentan contra la vida. Consideramos que es una burla a la propia cultura, a los usos y costumbres de los nahuas de la sierra de Zongolica. No es correcto, se están aprovechando de una ceremonia ritual para engañar a la gente, haciéndoles creer que es algo bueno, como si se tratara de algo que nos va a favorecer a los pobladores del lugar".

Finalizó:

"Ellos han buscado entre los pobladores a los que elaboran estos xochitlallis sin explicarles cuál sería el fin de esa labor; pero te repito: ellos, los que buscan no conocen la esencia, no comprenden la espiritualidad de esta costumbre. No vas a ir a dañar mortalmente a tu madre y justificarte diciendo que ya le pediste perdón" 30.

28 Enríquez, 2014.

29 Sánchez y Betancourt, 2011.

30 Chaín, 2013. 


\section{LA MOVILIZACIÓN Y LA RESISTENCIA INDÍGENA}

Ante la inminente construcción de la presa hidroeléctrica, integrantes de los pueblos indígenas nahuas de la Sierra de Zongolica han realizado diversas movilizaciones, mostrando su disconformidad. En estos actos acusan a la empresa Electricidad del Golfo por dejarles más pobreza y destrucción en sus sitios de origen: "violando nuestros derechos como pueblos indígenas, específicamente los plasmados en el artículo $5^{\circ}$ del convenio 169 de la Organización Internacional del Trabajo, el cual habla del derecho de los pueblos a ser consultados respecto a sus territorios", y que "Ios beneficios solo son para la empresa y provoca graves afectaciones a quienes menos tienen y que viven en estos municipios marginados. De hecho, puntualizan que este proyecto hidroeléctrico violó el derecho que tienen de vivir en paz, de tener libertad y seguridad" 31.

Las organizaciones regionales de Zongolica antes mencionadas - en un comunicado enviado a la prensa nacional, a las autoridades ejidales y al pueblo de México el 10 de julio de 2011_32 argumentaban que no se había consultado a las comunidades para que pudieran opinar sobre el proyecto; que no les habían informado de los beneficios ni de los daños que causaría; que las compras de tierras se hicieron con mentiras y prestanombres y que las empresas dejaban los daños a las comunidades y se iban. Por estas razones exigían a las autoridades competentes, estatales y federales, la cancelación del proyecto.

El 5 de junio de 2013 se organizó un foro en la región bajo el lema "Escuchemos la Palabra del Agua", a propósito del día mundial del medio ambiente, organizado por el Decanato de la sierra de Zongolica, Pastoral Indígena, la Universidad Veracruzana y también la organización Toaltepeyólotl, Asociación Civil pro derechos humanos, que señalaban:

"Hay muchas dudas, no se entiende en qué momento se autoriza la construcción de una represa privatizando un río, dejando sin agua a muchas comunidades que hacen uso de ese agua para bañarse, para acarrear agua para la casa, para lavar e incluso para los sembradíos, 0 sea, no se comprende pues esta situación" 33 .

El caso de la presa de Zongolica no está en silencio. Se ha denunciado sistemáticamente: en noviembre de 2012 los nahuas denunciaron la violación a sus derechos como pueblos ante el Tribunal Permanente de los Pueblos (TPP) y en 2014 se emitió un dictamen sobre el caso realizando recomendaciones al gobierno mexicano para que se visibilicen las violaciones de los derechos indígenas; en julio de 2013 en el Encuentro Nacional e Internacional de Afectados Ambientales señalaron que en la violación de derechos humanos están involucrados el gobierno federal, la Secretaría de Medio Ambiente y Recursos Naturales (Semarnat), la Comisión Reguladora de Energía, la Secretaría de Energía (SE), la Comisión Federal de Electricidad (CFE)... Y en el estado desde

31 Rodríguez, 2011. En esta movilización participan organizaciones regionales como la Unión de Ejidatarios y Comuneros del Valle de Tuxpango (UECVT); Timocepanotoke Noche Altepeme Macehualme, Unión de todos los pueblos pobres (TINAM) y el Frente Nacional de Lucha por el Socialismo (FNLS).

32 http:// www. kaosenlared. net/ noticia, 2013.

33 http:// zapateando. wordpress.com, 2011 el gobernador, la Comisión Estatal del Agua (CAEV), que han autorizado permisos con irregularidades, y los gobiernos municipales de Texhuacan, Zongolica y Mixtla de Altamirano, por no haber realizado consulta pública sobre el proyecto de la presa de Zongolica ${ }^{34}$.

Las organizaciones indígenas optan por acudir a tribunales internacionales como una alternativa para exigir al gobierno mexicano el cese a la violación de sus derechos como pueblos indígenas y es ahí donde seguramente encontrarán un mayor apoyo a sus demandas ${ }^{35}$.

\section{Efectos culturales de la obra en la población indígena}

Uno de los problemas más importantes derivados de este tipo de obras es la necesidad de restituir sus tierras a las poblaciones afectadas y la consecuente afectación a su cultura y tradiciones. Para los pueblos indígenas la tierra no representa solamente un medio de producción; un bien que pueda ser equitativamente intercambiado por otro bien de similar naturaleza. Como ámbito donde ha transcurrido la vida colectiva de un grupo humano, el espacio residencial queda ligado a la historia grupal. La geografía de una región se puebla así de significados que las sociedades depositan en ella, y que refieren a sucesos considerados claves de su trayectoria temporal. Esto es, desde la antigüedad hasta nuestros días, en los pueblos indígenas existe un equilibrio entre el hombre y la naturaleza a través de cuatro principios: lo mítico, lo sagrado, lo espiritual y lo cosmogónico, que permiten que exista una reciprocidad entre tierra-territorio. Por ello, es fundamental señalar que tierra y territorio no son lo mismo; el primero no sólo es la parcela, sino que en ella viven los pasados, los animales, las plantas y los seres que habitan en ella; el territorio, en cambio, es el espacio vital para desarrollar las actividades culturales, la economía, la política, lo social y lo sagrado (los rituales, los lugares sagrados, la fiesta). Existe pues, una estrecha vinculación entre la identidad cultural y la territorialidad indígena.

Así, estamos ante dos visiones del mundo y distintas visiones del desarrollo. Como ejemplo, veamos la concepción indígena y no indígena del agua y del territorio.

\footnotetext{
34 El Tribunal Permanente de los Pueblos es un tribunal ético, internacional y de carácter no gubernamental. Información en su página de internet indica que "examina las causas de la violación de los derechos fundamentales de los pueblos, determina si, en efecto, han sido violados tales derechos y, finalmente, denuncia ante la opinión pública internacional a los autores de dichas violaciones" (http:// www.tppmexico.org/).

35 La situación para los pueblos indígenas en México es crítica. Por ejemplo, en el caso de la presa El Naranjal, Veracruz, más de treinta comunidades se unieron -desde junio de 2011- para conformar el colectivo Defensa Verde, Naturaleza para Siempre. El Colectivo se ha dedicado a solicitar información al gobierno y han emprendido diversas acciones (marchas, pintado de bardas, pega de carteles y foros informativos y culturales). El colectivo lleva ya 32 asambleas. Entre sus demandas está la cancelación del proyecto y su consigna es mantener la defensa de la tierra y de los recursos naturales (más del setenta por ciento de los terrenos no los han vendido). Sin embargo, en 2013 uno de los líderes Noé Salomón Vázquez Ortiz fue asesinado a pedradas en Amatlán de los Reyes, un día antes de la reunión nacional de más de quinientos ambientalistas de diferentes regiones del país.
} 


\section{El agua}

De acuerdo a los pueblos indígenas, el agua es concebida como un ente sagrado. Por lo tanto, en ella viven diferentes seres que los apoyan en su vida. En cambio, la concepción que tienen del agua los no indígenas es la de un bien puramente comercial, se puede vender, rentar, desperdiciar e incluso modernizar, como es el caso de las presas hidrológicas e hidroeléctricas ${ }^{36}$.

Hasta antes de la construcción de la presa, los pueblos nahuas de la sierra contaban con una infinidad de manantiales, pozos y humedales que duraban todo el año, más aquellos que eran de temporal. Cada pozo, recibe anualmente rituales para que no se seque. La mayoría de los nahuas heredaron de su pasado el respeto y la reciprocidad del hombre con la naturaleza, por lo que los ritos de la costumbre hacia los pozos son muy importantes. Igualmente, el reconocimiento de la medicina tradicional, la realización de los ritos hacia los muertos, a las cosechas, a la lluvia para que no falte, incluso para que la furia de la naturaleza los proteja. Los rituales van dirigidos, por lo tanto, al agua, al fuego, al sol, a la tierra, entre otros.

A la tierra se le ofrenda pan, pollo, amigo (aguardiente), chile, café, cerveza... Se le ofrecen para que haya reciprocidad. En los pozos se realiza el ritual a principios de mayo (dos, tres, muchas veces). Hasta allí llega el curandero que entrega a la tierra lo que pide, se le reza, se le baila y la gente se queda toda la noche alrededor del pozo para que no falte agua. Antes se recorrían todos los pozos, ahora ya no.

\section{EL TERRITORIO O LA TERRITORIALIDAD}

Los nahuas de Zongolica viven en un territorio caracterizado por la abundancia de cerros, montañas, lomas, escarpes y cuevas que tuvieron y tienen, aún hoy en día, un significado con la madre tierra, asociada a la gestación, el nacimiento y la fertilidad, y otras deidades relacionadas con estos elementos de la naturaleza. Inicialmente debieron servir como refugio y sitio de habitación. Posteriormente, obtuvieron otras connotaciones más relacionadas con la religión y, por lo tanto, se convirtieron en boca 0 vientre de la tierra, entradas al inframundo, morada de los dioses del agua y los de la muerte. En no pocos casos estos espacios se convirtieron en lugares de culto que servían a ciertos rituales y en los que se debían dejar las ofrendas a las deidades, de modo que las cuevas constituían áreas sagradas del paisaje natural. Estas también sirvieron como un ámbito ideal para enterrar a los muertos.

Como se analizó en párrafos anteriores, el hombre nahua concibe que está en el centro del mundo o maxi mundo y este se encuentra delimitado por los cerros sagrados y por el mundo celeste y el inframundo. Este mundo, además, se encuentra vinculado a diferentes divinidades que son las que sostienen a los hombres y estos, a su vez, a ellas. Esta geografía simbólica contiene

36 La sierra de Zongolica tiene tres estaciones climatológicas durante el año: la época de lluvias, entre junio y octubre; luego viene una de lloviznas, neblinas, húmeda, fría con nortes y heladas, entre noviembre y febrero; y otra seca o menos húmeda de marzo a mayo.
Iugares que representan al gún hecho social o cultural. El espacio no es estático, es flexible, ya que en él interactúan naturaleza, dioses fundadores, lugares de origen, mitos, individuos, familia, comunidad, instituciones y antepasados de los nahuas actuales.

La religiosidad de los pueblos indígenas nahuas se encuentra en una cosmovisión ancestral sustentada en el sol, la luna, el agua, la tierra, el viento, el rayo, los cerros y el maíz, la coherencia básica para la consolidación de su ciclo de vida, la concepción de las enfermedades y su curación, el ciclo anual de sus actividades productivas y el calendario festivo. La visión del mundo y la cosmología de las sociedades indígenas se expresa cotidianamente en sus mitos y en sus ritos. A la par de la existencia de un mundo natural, para el pueblo indígena nahua existe un mundo de lo sobrenatural que explica y da sentido a aquel. Los cerros son la morada de los dioses ancestrales y las cuevas son los umbrales que separan al hombre del mundo de lo sagrado ${ }^{37}$.

La alteración de los elementos de su territorio afecta su vida social, económica y religiosa. En palabras de Cira Quechulpa Pérez, mujer indígena de la Sierra de Zongolica:

"Destruirle el rostro a la madre tierra de un solo jalón es doloroso.
Para nosotros, el haberle hecho un túnel hacia su interior es des-
truirle su corazón. Le destruyes su rostro, le quitas su ropaje, le
desgarras su vestido. La madre tierra es la que te da de comer, te
da el sustento, es la que te da la vida y te guarda cuando tú mue-
res. Estar en contra de la presa no es estar en contra del desarro-
Ilo. ¿Cómo concibes el desarrollo hacia las comunidades indígenas
cuando vienes y destruyes? No puedes construir sobre lo que estás
destruyendo" 38 .

Desde esta cosmovisión, pertenecer a la Madre Tierra es parte de la identidad indígena y está vinculada a la memoria de los pueblos, es algo vivo y con historia. Igualmente los saberes y conocimientos que se han acumulado de generación en generación son parte del territorio, así como los lugares sagrados, los recursos naturales y el tiempo, los ciclos agrícolas de cultivos sagrados son la base del ordenamiento del tiempo familiar y comunal, que es diferente en cada territorio.

En suma, los diferentes análisis arrojan elementos que ayudan a comprender el tema que nos ocupa. Por ejemplo, la perspectiva cultural nos permite entender la estrecha vinculación entre la concepción simbólica del mundo indígena con la identidad y la territorialidad de sus habitantes, así como con algunos rituales que se practican actualmente y que muestran la estrecha relación entre el hombre, los dioses, las deidades, el territorio y los lugares sagrados. Se puede decir que la geografía simbólica, como un concepto que integra el etnoterritorio, ayuda a explicar los lugares sagrados de los pueblos indígenas. Y en relación al marco jurídico de los derechos indígenas y el patrimonio cultural, aunque todavía es insuficiente el tratamiento de estos temas en la legislación actual tanto nacional como internacional, ya existen elementos para avanzar en el reconocimiento a los derechos indígenas, sus recursos naturales y territoriales.

\footnotetext{
37 Velasco, 2002
}

38 Sánchez y Betancourt, 2011. 
Tierra y Agua: efectos culturales por la construcción de la hidroeléctrica en los pueblos indígenas nahuas de Zongolica, Veracruz

\section{Reflexiones finales}

Como se señaló a lo largo del texto, México tiene una amplia historia en la construcción de presas de almacenamiento e hidroeléctricas con la consecuente afectación a comunidades y pueblos indígenas. Miles de personas han sido reubicadas en otros lugares y en todos los casos ha sido una constante la violación a los derechos de los pueblos indígenas. Se advierte que el modelo de desarrollo que está impulsando el estado mexicano no se detiene ante nada. Es indiferente ante la amenaza a nuestra biodiversidad, ante culturas y pueblos, ante cuestiones tan importantes como son los derechos humanos y sociales de sus habitantes. Esto lo podemos revisar en el informe del Fondo Mundial para la Naturaleza, donde menciona los siete errores capitales que realizan los gobiernos al construir presas, ignorando a la población local afectada ${ }^{39}$.

En el informe se comenta que si se construyen, planean y operan adecuadamente mejora la seguridad alimenticia y energética. Esto no se da en México, porque hay intereses financieros y políticos por encima de los derechos de las personas: estos intereses inhiben cualquier posibilidad de diálogo, transparencia y consulta con la población. Sin duda, los efectos que acarrean estas grandes obras son negativos, pues implican la reubicación de numerosas poblaciones, la destrucción de sus sitios ceremoniales y rituales. De hecho, no se toma en cuenta el territorio como un ente y no se valora la cultura. Es negativo que se sigan destruyendo áreas de alto valor ecológico sin tomar en cuenta la biodiversidad, la vocación económica y la forma de vida de las poblaciones.

Se demuestra cómo las instituciones gubernamentales involucradas en este tipo de obras se han valido de engaños. Solo han dejado promesas y no han respetado la legislación mexicana que, aunque de manera incipiente, establece el respeto y protección de los pueblos indígenas. También hemos visto como han ignorado los convenios internacionales firmados por México. Ninguno de los proyectos de presas ha seguido un buen proceso; por el contrario, todos han dejado problemáticas sin resolver. Para finalizar, sería de gran importancia que organismos internacionales presionen al estado mexicano a fin de que cumpla las leyes y convenios, además de resolver a favor de los pueblos indígenas, con el objeto de evitar que se sigan cometiendo los mismos errores.

\section{Bibliografía}

Barabas, Alicia M. 2001: "Territorialidad, santuarios y peregrinaciones", en Diario de Campo (Boletín interno de los investigadores del área de Antropología), 34, INAH, 16-18.

Barabas, Alicia M. y Bartolomé, M. A. 1992: "Antropología y Relocalizaciones", en Alteridades, 2, 4, México, 5-15.

Chaín Revuelta, Francisco J.: "La Madre tierra no les dio permiso después del Xochitlali", en http://grandesmontanas1106.blogspot. $\mathrm{mx} / 2011$ / 06/ la-madre-tierra-no-les-dio-permiso. html (consulta realizada el 27 de marzo de 2013).

\footnotetext{
39 Enciso, 2013.
}

De Certeau, M. 2000: La invención de lo cotidiano. I. Artes de Hacer. México, Universidad Iberoamericana, Instituto Tecnológico y de Estudios Superiores de Occidente.

Descola, P. 2001: "Construyendo naturalezas, ecología simbólica y práctica social", en Descola, P. y Pálsson, G. (coords.), 2001: Naturaleza y sociedad. Perspectivas antropológicas. México, Siglo XXI, 101-123.

Enciso L., Angélica, 2013: "La construcción de presas descuida la biodiversidad e ignora la comunidad. Presenta el Fondo Mundial para la Naturaleza informe", en La jornada, 28 de marzo de 2013, 35 (consulta realizada el 3 de junio de 2013).

Giménez, G. 2000: "Territorio, cultura e identidades: la región sociocultural", en Rosales Ortega, R. (coord.): Globalización y regiones en México. México, UNAM-Porrúa.

Haesbaert, R. 2004: 0 mito da desterritorializaçao: do "fim dos territórios" a multiterritorialidade. Rio de J aneiro, Bertrand Brasil.

HIC-AL, 2010: Presa Cerro de Oro, 35 años sin justicia. México.

HIC-AL, 2012: Dictamen final del jurado de la Pre-audiencia "Presas, derechos de los pueblos e impunidad", Mesa de devastación ambiental y derechos de los pueblos, Tribunal Permanente de los Pueblos (TPP) - Capítulo México, de la Oficina de Coordinación Regional para América Latina de la Coalición Internacional para el Hábitat (disponible en http:// www. hic-al. org/ noticias. cfm?noticia=1306).

Hoffman, O. y Salmerón Castro, Fernando I. (coords.) 1977: "Introducción. Entre representación y apropiación, las formas de ver y hablar del espacio", en Nueve estudios sobre el espacio. Representación y formas de apropiación, México, CIESAS, 13-29.

http:// sucesosdeveracruz.com.mx, 2014: "Hidroeléctrica de Zongolica; Compromisos sin cumplir", por J uan J osé Enríquez, en Sucesos de Veracruz, 23 de septiembre de 2014 (en http:/ / sucesosdeveracruz. com. $\mathrm{mx} /$ reportaj es/ otros/452-hidroelectrica-de-zongolica-compromisos-sin-cumplir.html).

http:// www. kaosenlared. net/ noticia/ 174815/ mexico-rechazan-presas-hidroelectricas-zongolica-valle-tuxpango (consulta 13 de agosto de 2013)

http:// www. sinembargo.mx, 2012: "Indígenas de Nayarit piden analizar contaminación en presa Aguamilpa" (28 de marzo de 2012), en http:// www. sinembargo. mx/ 28-03-2012/ 192367 (consulta 4 de mayo de 2013).

http:/ / zapateando. wordpress. com/ 2011/ 07/ 13/ presa-hidroelectricaafecta-mixtla-de-altamirano-san-juan-texhuacan-y-zongolica-privatizaron-el-rio-dejando-sin-agua-a-comunidades-nahuas (consulta 3 enero de 2013).

http:// zapateando. wordpress.com: "Presa Hidroeléctrica afecta Mixtla de Altamirano, San J uan Texhuacan y Zongolica. Privatizaron el río dejando sin agua a comunidades nahuas: Toaltepeyolotl, 2011" (en http:// zapateando. wordpress. com/ 2011/ 07/ 13/ presa-hidroelectrica-afecta-mixtla-de-altamirano-san-juan-texhuacan-y-zongolica-privatizaron-el-rio-dejando-sin-agua-a-comunidades-nahuas, consulta 13 de agosto de 2013).

INEGI (Instituto Nacional de Estadística y Geografía), 2010: Censo de Población y Vivienda.

Iracheta-Cenecorta, M. P. y Gómez-Marcial, B. L. 2014: "Sembramos por necedad". La Central Hidroeléctrica Zimapán y el sector agrícola en las localidades de Bellavista del Río y La Mora del Municipio de Cadereyta de Montes, Querétaro, México, 1960-2010", en Agua y Territorio, 4, 35-46. http:// dx. doi.org/10.17561/at.v1i4. 2162 
Pérez U., M. 1996: "Hasta 1993, el INI no sabía de la existencia de los mayos de Huites", en La J ornada, 21 de junio de 1996 (www.jornada. unam. mx/ 1996/ 06/21/ presa2.txt.html).

Rodríguez, María T. 2003: Ritual, identidad y procesos étnicos en la sierra de Zongolica. México, CIESAS.

Rodríguez Hernández, M. 2011: "Indígenas de la Sierra de Zongolica marcharon en Orizaba contra presa hidroeléctrica", en http:// www. alcalorpolitico.com/ informacion/ indigenas-de-la-sierra-de-zongolica-marcharon-en-orizaba-contra-presa-hidroelectrica-72476. html\#. V6CO02MsCg8 (20 de junio de 2011).

Sainz, G., 2013: La Hidroeléctrica El Naranjal. Colectivo de Defensa Verde, Naturaleza para siempre.

Sánchez, I. y Montalvo Méndez, M. 2012: "Presas hidroeléctricas: un modelo de imposición, despojo y promesas incumplidas", en http:// desinformemonos.org (4 de noviembre de 2012, consultado el 2 de mayo de 2013).
Sánchez, M. y Betancourt, R. D. 2011: "Gobierno autoriza hidroeléctrica privada sin consultar a indígenas", en Contra línea, 246, 17 de agosto de 2011 (consulta 23 de noviembre de 2012).

Scott S. Robinson, 2012: "Megaproyectos: presas, minas y demás", en La Jornada del Campo, 57, 23 de junio 2012 (en www.jornada. unam. mx/2012/06/23/cam-minas.html, consulta 7 de mayo de 2015).

Tribunal Permanente de los Pueblos: Movimiento mexicano de Afectados por las Presas y en Defensa de los ríos. Pre audiencia Presas, Derecho de los pueblos e impunidad, 2012

Tribunal Permanente de los Pueblos: La Construcción de presas en México, Jalisco, 2012, p. 2 en http:// pasodelareina.org/wp-content/ uploads/ 2013/ 03/ LA-CONSTRUCCI\%33\%33N-DE-PRESAS-ENM\%3\%89XICO. pdf. Consulta realizada el 22 de noviembre de 2012.

Velasco Toro, J. 2002: El péndulo de la resistencia (La defensa de la territorialidad y autonomía indígena). Veracruz, Fondo Editorial de Culturas Indígenas. 